\title{
Further Consideration of Lymphadenectomy Along the Left Recurrent Laryngeal Nerve During Robot-Assisted Minimally Invasive Esophagectomy
}

\author{
Kazuto Harada, MD, PhD, Masaaki Iwatsuki, MD, PhD, Yoshifumi Baba, MD, PhD, Naoya Yoshida, MD, PhD, and \\ Hideo Baba, MD, PhD
}

Department of Gastroenterological Surgery, Graduate School of Medical Science, Kumamoto University, Kumamoto, Japan

The lymph nodes (LNs) along the recurrent laryngeal nerves are among the LNs most frequently involved in esophageal squamous cell carcinoma; therefore, these LNs should be completely dissected. However, injury to the recurrent laryngeal nerves during $\mathrm{LN}$ dissection can cause palsy, with consequent aspiration pneumonia postoperatively. Moreover, this complication results in unfavorable postoperative survival. ${ }^{1}$ Several operative techniques have been described to ensure safe and high-quality dissection of the LNs along the left recurrent laryngeal nerve. During esophagectomy, the anatomical layers should generally be divided based on embryologic foregut development. Several concepts regarding the membranous structures in the upper mediastinum, such as the visceral sheath and mesotracheoesophagus, have been reported. ${ }^{2,3}$ Oshikiri et al. $^{4}$ described a procedure for dissecting the LNs along the left recurrent laryngeal nerve by minimally invasive esophagectomy (MIE) and termed this the 'Bascule method'. After mobilizing the upper esophagus from the trachea by cutting the tracheal arteries and then mobilizing the tissue together with the left recurrent laryngeal nerve and LNs from the trachea, the so-called "esophageal

This work has not been previously presented to a society or meeting.

(C) Society of Surgical Oncology 2021

First Received: 24 February 2021

Accepted: 2 March 2021;

Published Online: 17 July 2021

H. Baba, MD, PhD

e-mail: hdobaba@kumamoto-u.ac.jp mesenteriolum' can be recognized. The Bascule method enables simple recognition of the esophageal mesenteriolum by dividing the esophagus and then drawing the proximal side of the divided esophagus.

More recently, Oshikiri ${ }^{5}$ developed the "modified Bascule method' by robot-assisted MIE (RAMIE). In this novel approach, Oshikiri ${ }^{5}$ proposed that RAMIE using articulated robot arms facilitates division and discernment of the following three layers: layer A, consisting of autonomous nerve branches from the right thoracic sympathetic ganglion; layer B, consisting of the thoracic duct and autonomous nerve branches from the left thoracic sympathetic ganglion; and layer $\mathrm{C}$, consisting of the left recurrent laryngeal nerve and LNs along the nerve. Oshikiri $^{5}$ showed that even after LN dissection, the left recurrent laryngeal nerve is still attached to the residual layer $\mathrm{C}$. This indicates that the recurrent laryngeal nerve can be protected from being touched and stretched. However, Oshikiri ${ }^{5}$ also reported that the rate of left recurrent laryngeal nerve palsy was $19 \%$ with this new method. All of the left recurrent laryngeal nerve palsies were defined as type I according to the Esophagectomy Complications Consensus Group definitions. ${ }^{6}$ Intraoperative neural monitoring might help to reduce the frequency of left recurrent laryngeal nerve palsy, especially type I palsy. ${ }^{7}$

According to the study by Oshikiri, ${ }^{5}$ RAMIE provides three important perspectives for future surgical practice. First, because the articulated robot arms enable performance of the operative procedure from many directions within a limited space, new surgical approaches that had previously been impossible in MIE can be performed using RAMIE. Second, the clearer view obtained by RAMIE might provide new insights into surgical anatomy. Finally, 
the fixed view and smooth movement without shakiness in RAMIE enable a more delicate procedure. The modified Bascule method is one of the novel techniques that can be performed using RAMIE. We hope that new technologies lead to the development of new methods or concepts, allowing RAMIE to improve patients' quality of life and prognosis after esophagectomy.

The goal of dissecting the LNs along the left recurrent laryngeal nerve is the avoidance of palsy and recurrence. However, whether RAMIE is superior to MIE with respect to postoperative morbidity, recurrent laryngeal nerve palsy, and the patient's prognosis remains unclear. The number of RAMIE procedures performed to date is limited-we are still in the learning phase. Oshikiri ${ }^{5}$ suggests that the modified Bascule method is an optimal standardization technique for surgeons who are in the learning phase of RAMIE. We hope that this technique will rapidly spread worldwide and contribute to the development of RAMIE.

In conclusion, the paper by Oshikiri ${ }^{5}$ suggests the possibility of the novel modified Bascule method for RAMIE. Further prospective studies are warranted.

ACKNOWLEDGEMENT The authors thank Angela Morben, DVM, ELS, from Edanz Group (https://en-author-services.edanz.co $\mathrm{m} / \mathrm{ac}$ ), for editing a draft of this manuscript.

FUNDING No sources of funding were used to assist in the preparation of this Editorial.

DISCLOSURE Kazuto Harada, Masaaki Iwatsuki, Yoshifumi Baba, Naoya Yoshida, and Hideo Baba have no conflicts of interest to declare.

\section{REFERENCES}

1. Baba Y, Yoshida N, Shigaki H, et al. Prognostic impact of postoperative complications in 502 patients with surgically resected esophageal squamous cell carcinoma: a retrospective single-institution study. Ann Surg. 2016;264(2):305-11.

2. Tokairin Y, Nakajima Y, Kawada K, et al. Histological study of the thin membranous structure made of dense connective tissue around the esophagus in the upper mediastinum. Esophagus. 2018;15(4):272-80.

3. Akagawa S, Hosogi H, Yoshimura F, Kawada H, Kanaya S. Mesenteric excision for esophageal cancer surgery: based on the concept of mesotracheoesophagus. Int Cancer Conf J. 2018;7(4):117-20.

4. Oshikiri $\mathrm{T}$, Yasuda $\mathrm{T}$, Harada $\mathrm{H}$, et al. A new method (the "Bascule method") for lymphadenectomy along the left recurrent laryngeal nerve during prone esophagectomy for esophageal cancer. Surg Endosc. 2015;29(8):2442-50.

5. Oshikiri T, Takiguchi G, Urakawa N, et al. Novel "Modified Bascule method" for lymphadenectomy along the left recurrent laryngeal nerve during robot-assisted minimally invasive esophagectomy. Ann Surg Oncol. https://doi.org/10.1245/s10434021-09738-w.

6. Low DE, Alderson D, Cecconello I, et al. International consensus on standardization of data collection for complications associated with esophagectomy: esophagectomy complications consensus group (ECCG). Ann Surg. 2015;262(2):286-94.

7. Kobayashi H, Kondo M, Mizumoto M, Hashida H, Kaihara S, Hosotani R. Technique and surgical outcomes of mesenterization and intra-operative neural monitoring to reduce recurrent laryngeal nerve paralysis after thoracoscopic esophagectomy: a cohort study. Int J Surg. 2018;56:301-6.

Publisher's Note Springer Nature remains neutral with regard to jurisdictional claims in published maps and institutional affiliations. 